\title{
Evaluating the Performance of Wireless Network using OPNET Modeler
}

\author{
Zainab T. Alisa, PhD. \\ Iraq, Baghdad \\ Baghdad University \\ Electrical Engineering Department
}

\begin{abstract}
Wireless networks have biggest constraint of limited bandwidth in comparison to wired networks but in spite of this constraint wireless networks are becoming popular day by day on account of their flexibility, mobility as well as inexpensive physical medium (air). This paper aims to simulate wireless local area network. The performance of the network is evaluated using optimized network engineering tool, OPNET 14.5 modeler,over four major physical layer technologies Infra-Red, Direct Sequence Spread Spectrum DSSS, Frequency Hopping Spread Spectrum FHSS and Orthogonal Frequency Division Multiplexing OFDM at multiple transmissionrates, $1 \mathrm{Mbps}, 2 \mathrm{Mbps}, 5.5 \mathrm{Mbps}, 6 \mathrm{Mbps}$, $9 \mathrm{Mbps}, 11 \mathrm{Mbps}$, 36Mbps,48Mbps, 54Mbps. DSSS can use $1,2,5.5,11 \mathrm{Mbps}$ rates. FHSS and IR are able to operate at 1or 2 Mbps rates, while OFDM is capable to operate at 6, $9,11,36,48$ or 54 Mbps rate.A trade-off exists between the selected data rate and the physical technology. It is founded that at some transmission rate, the OFDM technology delay is better than in DSSS. FHSS delay is less than DSSS delay. IR delay is better than FHSS and DSSS delay. In addition, for one physical layer technology the delay can be reduced by increasing the transmission rate of the channel.
\end{abstract}

\section{General Terms}

Throughput, delay, loads.

\section{Keywords}

Wireless LAN, IEEE 802.11 OPNET.

\section{INTRODUCTION}

The field of wireless local area networks (WLANs) is being widely studied and used in various emerging research domains such as mobile and pervasive computing, where WLANs provide high-speed wireless connection and support accessing information from anywhere and anytime. WLANs[1-3] support a wide range of applications, which may include simple applications such as web browsing, file transferring, etc. and the other ones, for instance, real-time multimedia applications (e.g., video streaming and video conferencing). IEEE 802.11is a vital standard for wireless LAN, which adopts the standard 802 logical link control (LLC) protocol that is further divided into two sub layers: physical layer (PHY) and medium access control (MAC) layer. This configuration provides optimized functionality for wireless communication. Initially 802.11 had two physical layers: Direct Sequence Spread Spectrum (DSSS) and Frequency Hopping Spread Spectrum (FHSS) and later, the physical layer have been categorized into three types with different physical characteristics and frequency spectrum. The physical characteristic of 802.11a and 802.11g are identical both are based on OFDM and support data rate of $54 \mathrm{Mbps}$.
However they differ in operating frequency spectrum802.11a operates on $5 \mathrm{GHz}$ band, while $802.11 \mathrm{~g}$ on $2.4 \mathrm{GHz}$. 802.11 bis based on DSSS and operates at $2.4 \mathrm{GHz}$ band with transmission rate from 1 to $11 \mathrm{Mbps}$. 802.11a has significant advantage due to the wide range spectrum of $5 \mathrm{GHz}$, having more number of independent channels. Both $802.11 \mathrm{~b}$ and $802.11 \mathrm{~g}$ are compatible with each other as both operates on $2.4 \mathrm{GHz}$ spectrum, but this may cause degradation in system performance as $2.4 \mathrm{GHz}$ is a small band spectrum with a lesser number of independent channels. The main objective of the work presented in this paper is to evaluate the performance ofwireless local area networkespecially end-toend delay, media access delay, load, traffic received and traffic sent.Infra-Red, Direct sequence, frequency hopping and OFDM has been utilized with different settingfor comparison between them. The network setuphave been simulated using OPNET 14.5 modeler. The simulation consists of sixteen scenarios. One scenario differs from the other scenario either in the physical layer technology or (and) transmission rate or (and) in data generation rate.

\section{RELATED WORK}

There exists a large body of research on wireless network, such as [4, 5, 6, and 7]. In [4], performance analysis of the Wireless and Wired computer networks through simulation has been attempted using OPNET as simulating tool.

The authors [5] simulated wireless LAN standard through the delivery of video traffic. The authors [6] suggest that Wireless LAN performance can be improved by fine tuning parameters such as fragmentation threshold, and buffer size. In [7], performance of 802.11 WLAN scenarios have been evaluated in OPNET Modeler 14.5.Throughput of the WLAN is evaluated in the presence of high priority traffic as well as low priority traffic, generating data simultaneously

\section{EXPERIMENTAL SETUP}

In order to study the performance of wireless networks, the network setup have been simulated using OPNET-14.5 simulator and various tests on it have been conducted. Performance of the proposed system is evaluated with different scenarios. The proposed network is an ad hoc network (Fig.1) consists of two independent wireless workstations (mobile). 


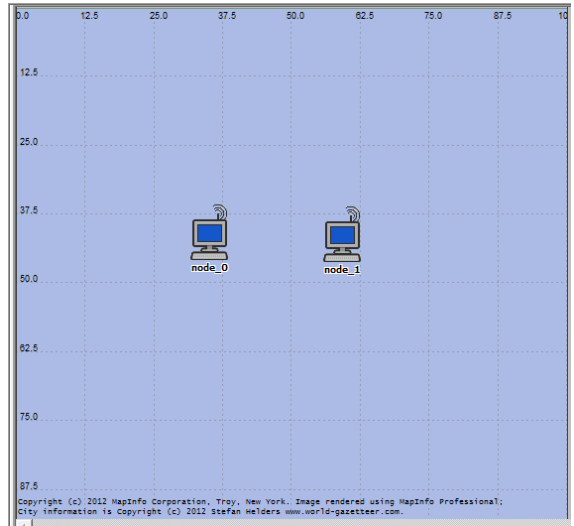

Fig.1: The Proposed Network

Tablelincludes the traffic generation parameters and Table 2 includesWLAN parameters for this work. The traffic generation parameters are selected such thatthe generation rate of data is $1 \mathrm{Mbps}$. The results are shown in figures (2-19)

Table 1.Traffic Generation Parameters.

\begin{tabular}{|l|l|}
\hline Attribute & Value \\
\hline Start Time (seconds) & Constant (10) \\
\hline On State Time (seconds) & Constant (600) \\
\hline Off State Time (seconds) & Constant (0) \\
\hline Interval Time (seconds) & 0.004 \\
\hline Packet Size (bytes) & 512 \\
\hline Stop Time (seconds) & Never \\
\hline
\end{tabular}

Table 2. WLAN Parameters

\begin{tabular}{|l|l|}
\hline Attribute & Value \\
\hline BSS Identifier & Auto Assigned \\
\hline Access Point Functionality & Disabled \\
\hline Physical Characteristics & $\begin{array}{l}\text { Infra-Red, Direct Sequence, } \\
\text { FrequencyHopping,OFDM }\end{array}$ \\
\hline Data Rate & $\begin{array}{l}\text { 1Mbps, 2Mbps, 5.5 Mbps, } \\
\text { 6Mbps, 9Mbps, 11Mbps, } \\
\text { 36Mbps, 48Mbps, 54Mbps }\end{array}$ \\
\hline Chanel Setting & Auto Assigned \\
\hline Buffer Size (bits) & 2560000 \\
\hline
\end{tabular}

\subsection{WLAN Delay}

Global statistics of WLAN delay is gathered for ten minutes of simulation time and can be observed in the following figures. WLAN delay represents the end to end delay of all the packets received by the wireless LAN MACs of all WLAN nodes in the network and forwarded to the higher layer.This delay includes medium access delay at the source MAC, reception of all the fragments individually, and transfers of the frames via AP (Access Point), if access point functionality is enabled. In this system the AP functionality is disabled as shown in table 2. WLAN end to end delay can be shown in figures (2-7).

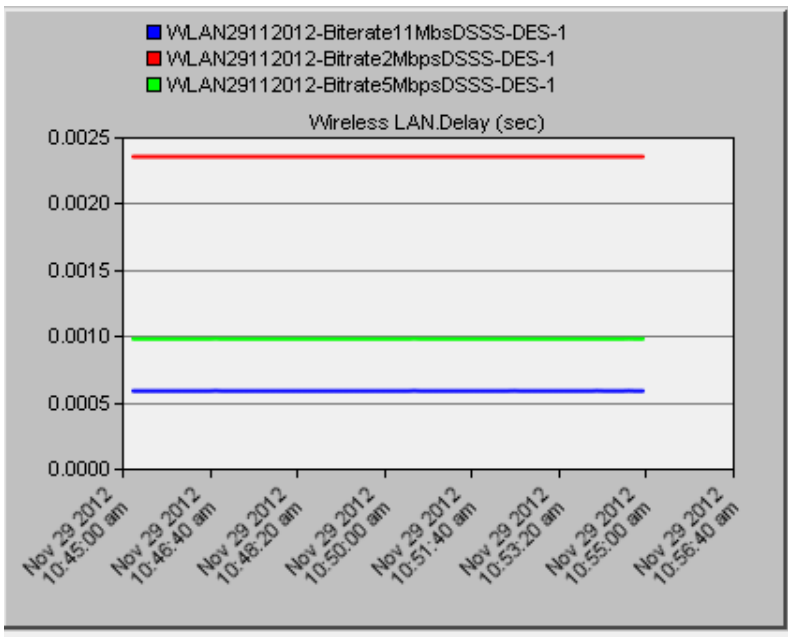

Fig.2: WLAN End to End Delay with DSSS Physical Layer Technology.

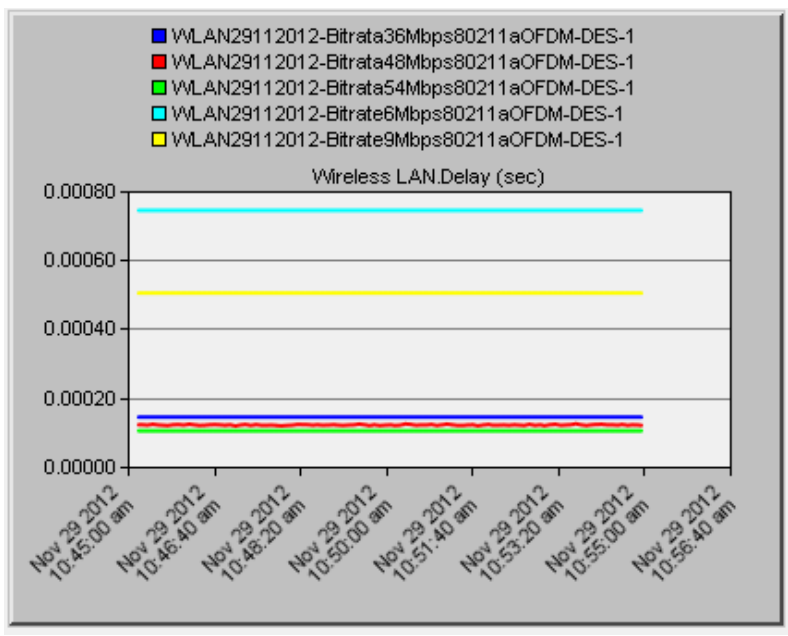

Fig 3: WLANEnd to End Delay with OFDM Physical Layer Technology.

As expected, the end to end delay is reduced with increasing the bit rate (fig. 2,3).A comparison between the end to end delays of different physical layer technologies at the same bit rate can be shown in figures $(4,5,6)$. Figure 6 shows the WLAN end to end delay for most scenarios presented in this paper. From these figures, it can be concluded that, at the same bit rate, the end to end delay in DSSS physical layer technology is greater than in OFDM and frequency hopping technologies. In addition, the figures show that thedelay for IR is lower than delays for DSSS and FHSS. The best delay can be obtained when the OFDM physical layer technology at $54 \mathrm{Mbps}$ is utilized. 


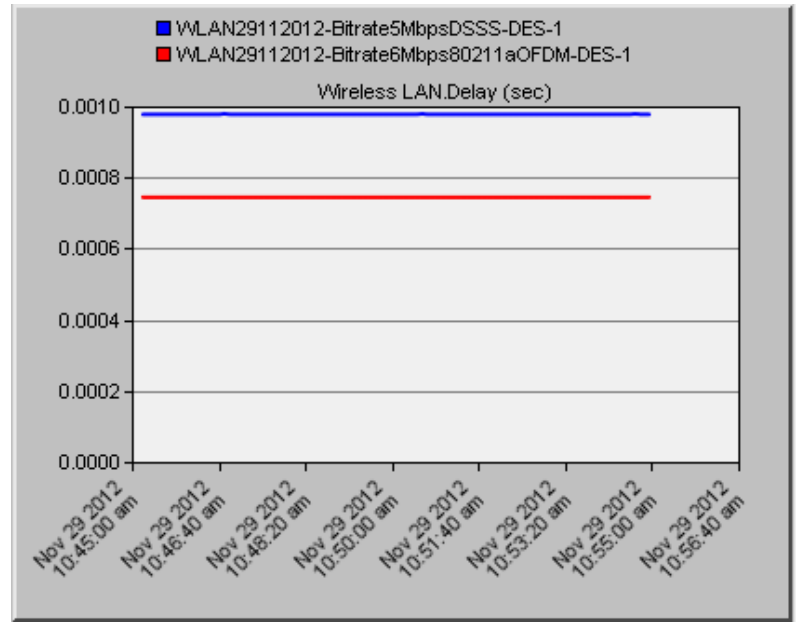

Fig.4: WLAN End to End Delay (DSSS at 5.5Mbps, OFDM at 6Mbps)

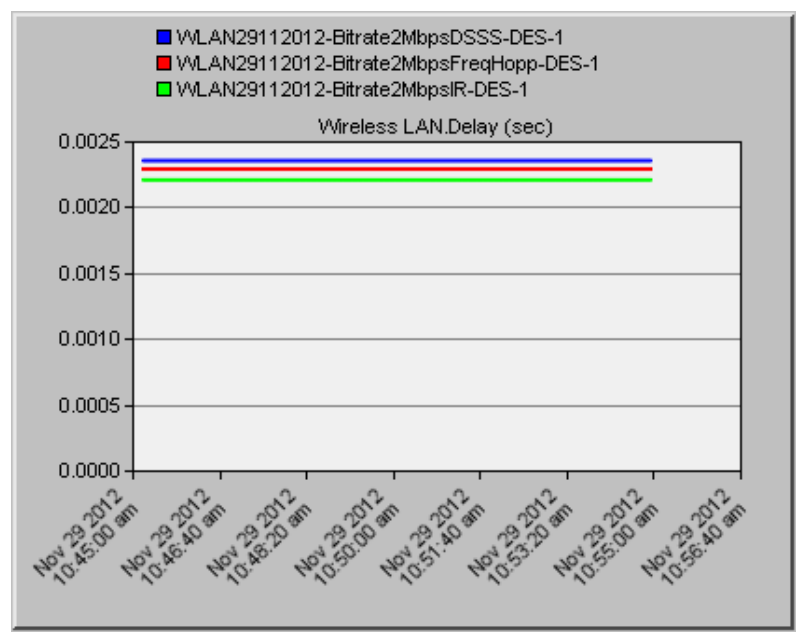

Fig.5:WLAN End to End Delay (DSSS at 2Mbps, Frequency Hopping at 2Mbps, IR at 2Mbps)

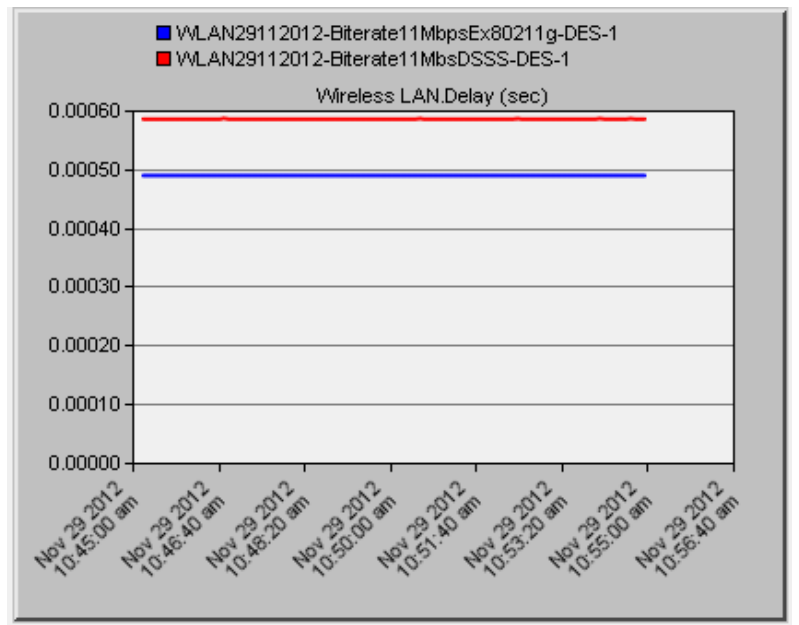

Fig.6: WLAN End to End Delay (DSSS at 11Mbps, Ex802.11g at 11Mbps)

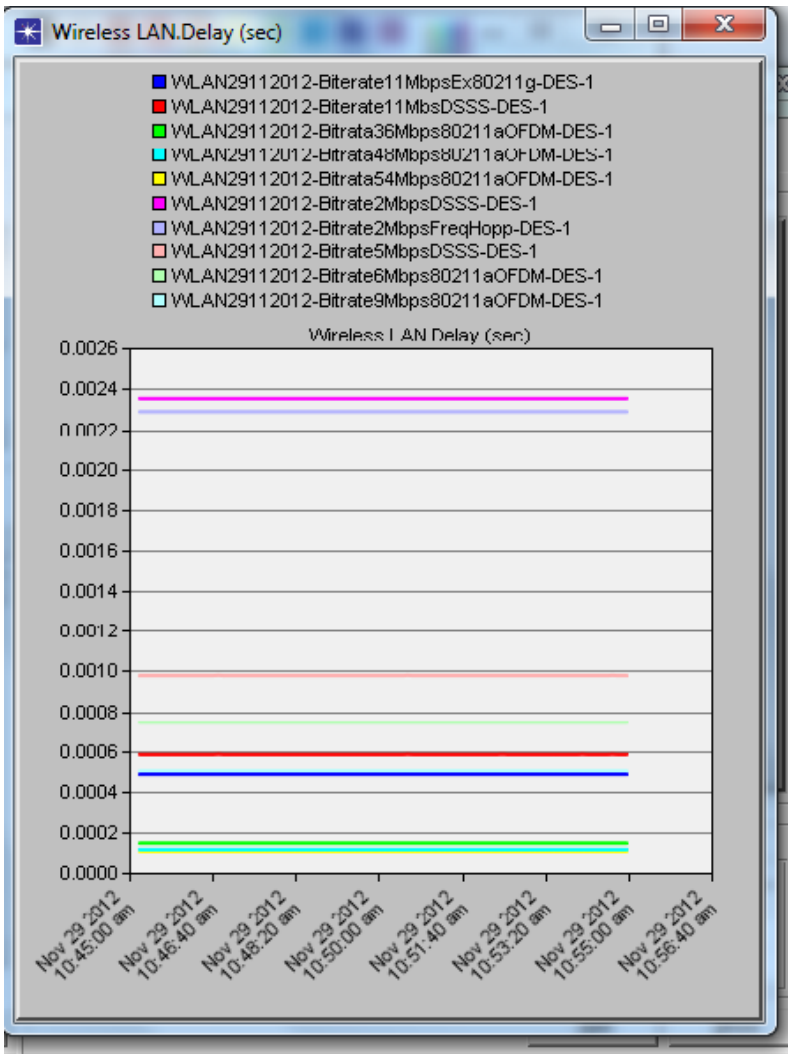

Fig 7: WLAN End to End Delay for the Proposed Scenarios

\subsection{Media Access Delay}

Media access delay represents the global statistic for the total of queuing and contention delays of the data, management, delayed Block-ACK and Block-ACK Request frames transmitted by all WLAN MACs in the network. For each frame, this delay is calculated as the duration from the time when it is inserted into the transmission queue, which is arrival time for higher layer data packets and creation time for all other frames types, until the time when the frame is sent to the physical layer for the first time. Hence, it also includes the period for the successful RTS (Request To Send)/CTS (Clear To Send) exchange, if this exchange is used prior to the transmission of that frame. Similarly, it may also include multiple numbers of backoff periods, if the MAC is $802.11 \mathrm{e}-$ capable and the initial transmission of the frame is delayed due to one or more internal collisions. This delay can be compared with the WLAN end to end delay.As shown in fig. (8-10) the media access delay can be neglected in compared with the end to end delay. 


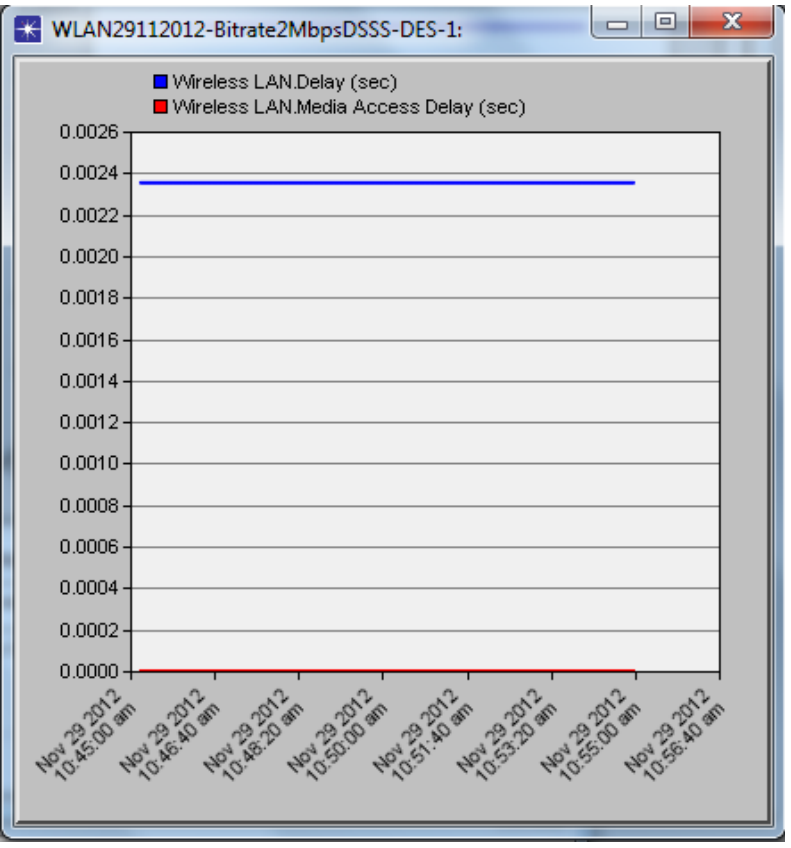

Fig.8: Media Access Delay and End to End Delay with DSSS Physical Layer Technology.

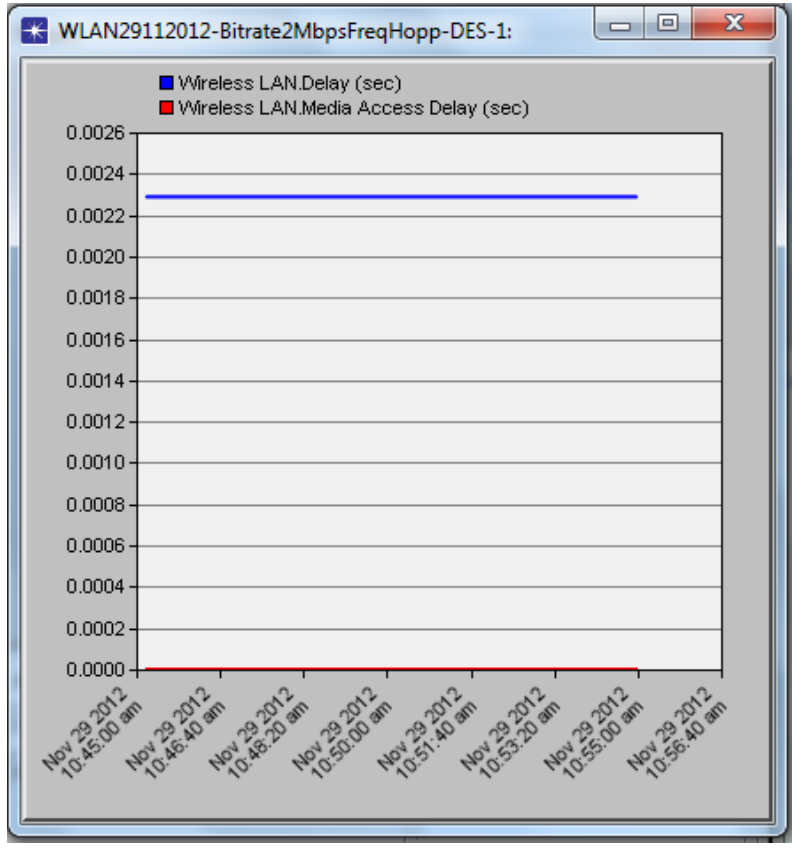

Fig.9: Media Access Delay and End to End Delay with Frequency Hopping Physical Layer Technology.

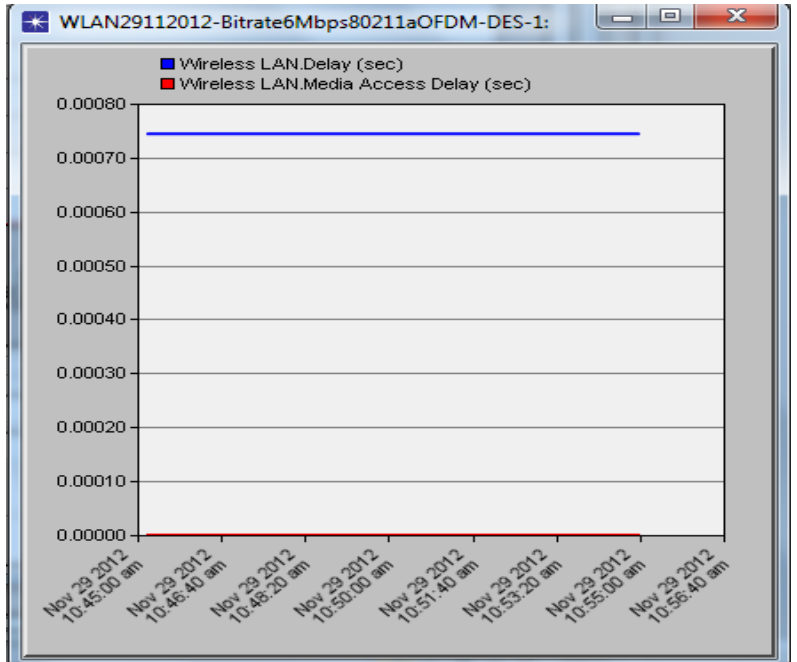

Fig.10: Media Access Delay and End to End Delay with OFDM Physical Layer Technology

\subsection{Traffic Received, Traffic Sent, Data Dropped, Throughput and Load}

The traffic received, traffic sent, data dropped,throughput and load are shown in figures (11-19).

The traffic generation parameters for the proposed system are selected in a way such that the generation rate of data is $1 \mathrm{Mbps}$. So the throughput, load, traffic received and traffic sent are $1 \mathrm{Mbps}$ for the proposed system and no dropped data as shown in figures (11-13). In the network with $1 \mathrm{Mbps}$ bite rate (fig.15), there is dropping in the data. The load on the network equal to $1 \mathrm{Mbps}$ but the throughput is less than this value (fig.14). The traffic receivedcan be improved (fig.16) by tuning the traffic generation parameters.

The load on the network is fixed for the proposed scenarios. So to improve the throughput of the system, the traffic generation parameters are tuned (fig.17\&18). The WLAN delay has been increased as shown in fig. 19 .

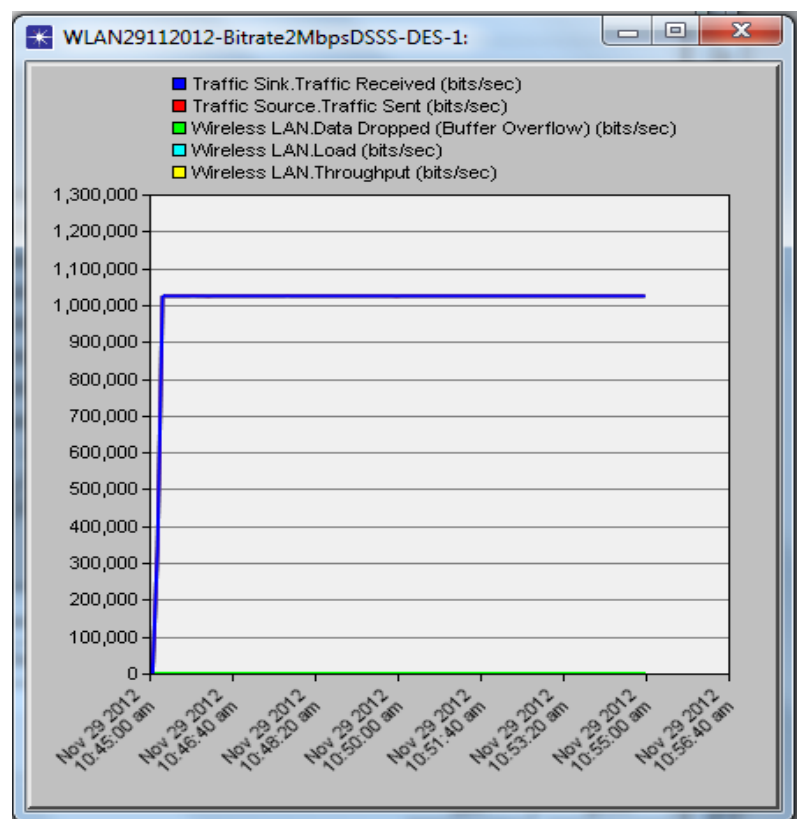

Fig.11: Traffic, Load, Throughput, Data Dropped for DSSS physical layer technology at 2 Mbps 


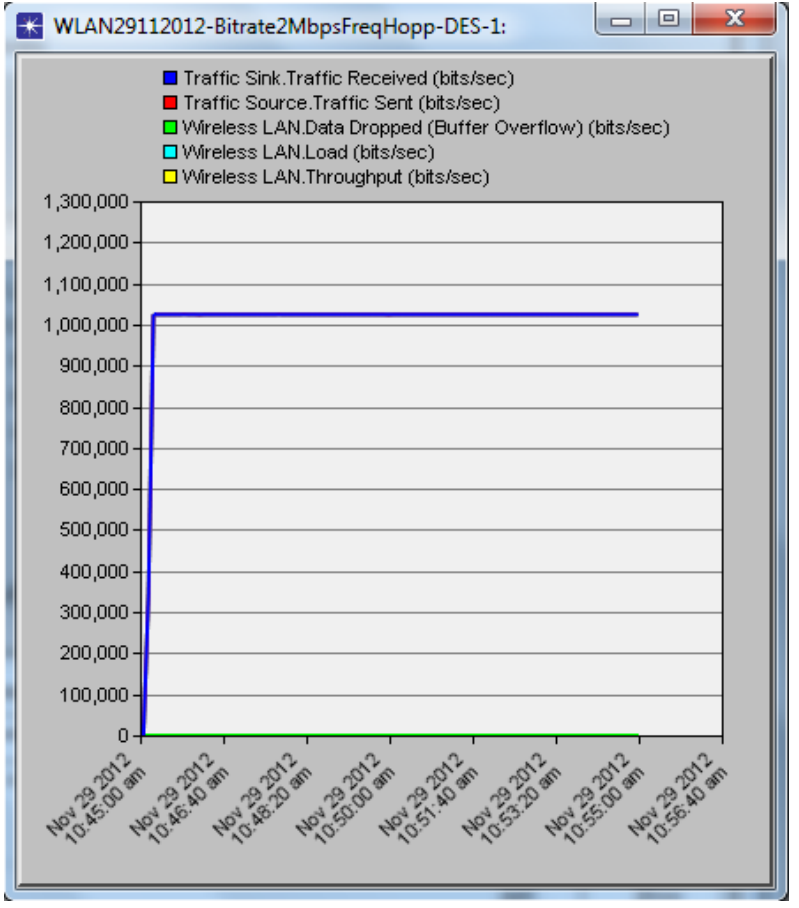

Fig.12: Traffic, Load, Throughput, Data Dropped for Frequency Hopping physical layer technology at 2 Mbps

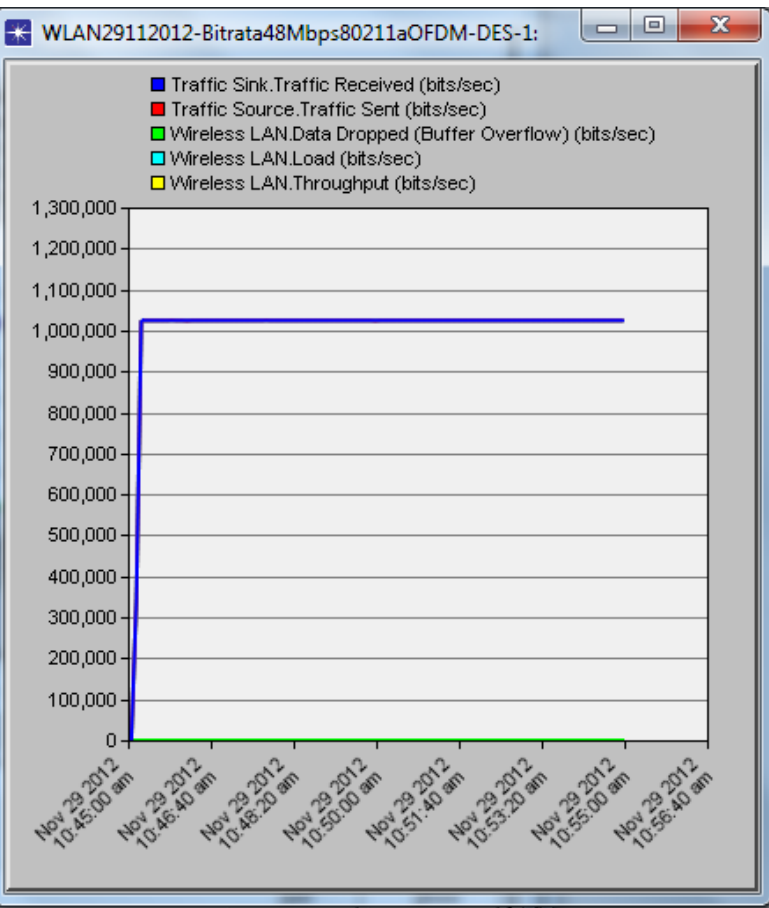

Fig.13: Traffic, Load, Throughput, Data Dropped for OFDM physical layer technology at $48 \mathrm{Mbps}$

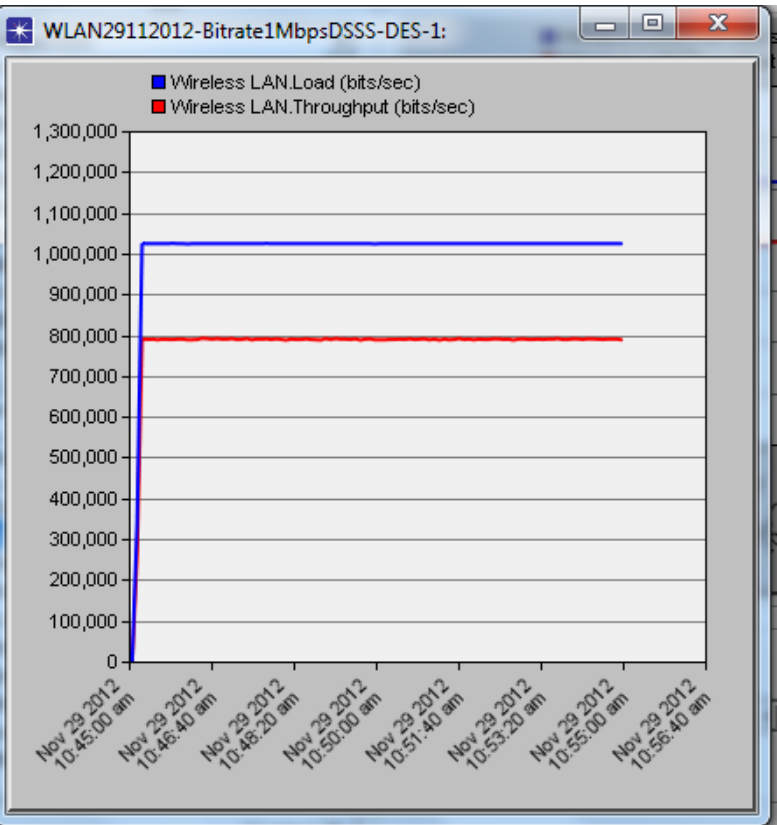

Fig.14: Load, Throughput for DSSS physical layer technology at 1 Mbps

\begin{tabular}{l|l|l|l|} 
WLAN29112012-Bitrate1MbpsDSSS-DES-1: & ㅁ & 回 & $\mathbf{X}$ \\
\hline
\end{tabular}

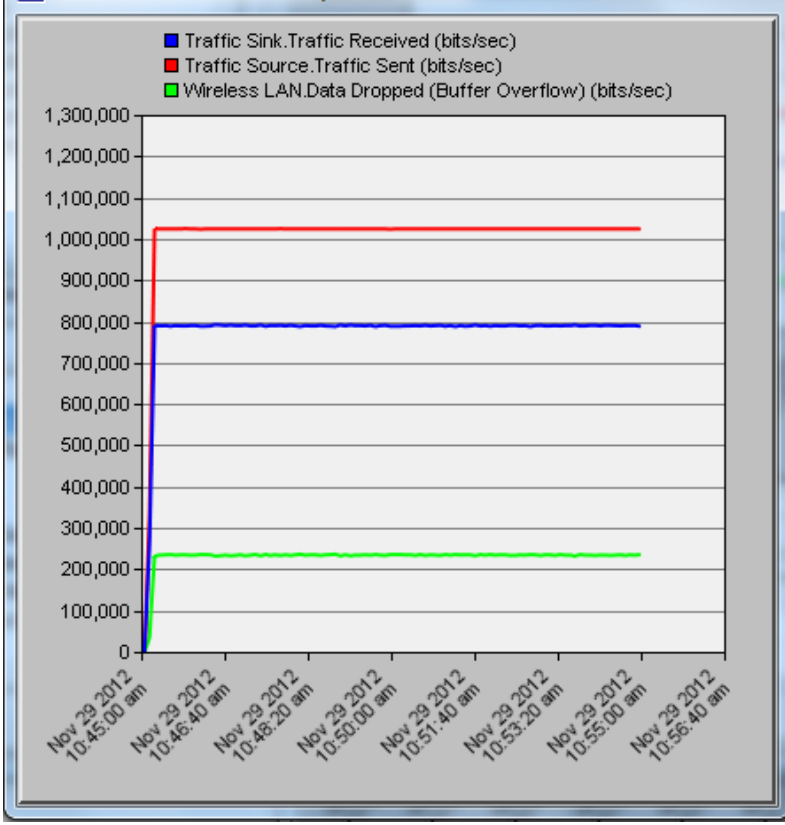

Fig.15:Traffic Received, Traffic Sent, Data Dropped for DSSS physical layer technology at 1 Mbps 


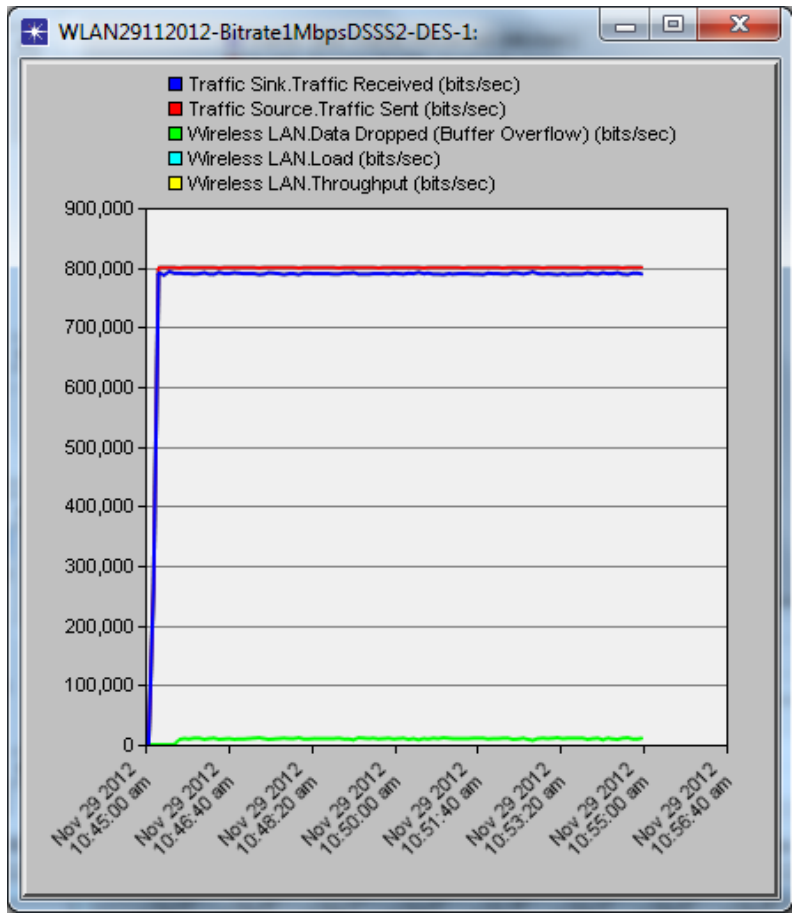

Fig.16:Traffic Received, Traffic Sent, Data Dropped, Throughput, Load for DSSS physical layer technology at $1 \mathrm{Mbps}$ after tuning traffic generation parameters

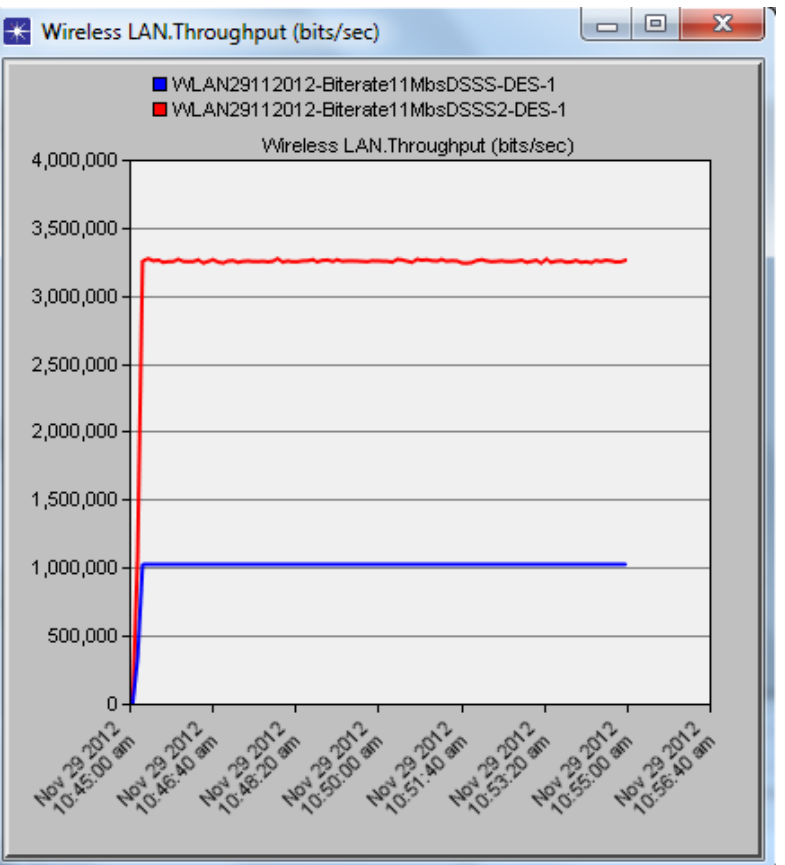

Fig.17: Throughputfor DSSS physical layer technology at 1Mbps before (DSSS) and after (DSSS2) tuning the traffic generation parameters
F. Wireless LAN.Load (bits/sec)

- MLAN29112012-Biterate11 MbsDSSS-DES-1

口MLAN29112012-Biterate11 MbsDSSS2-DES-1

Wireless LAN.Load (bitsisec)

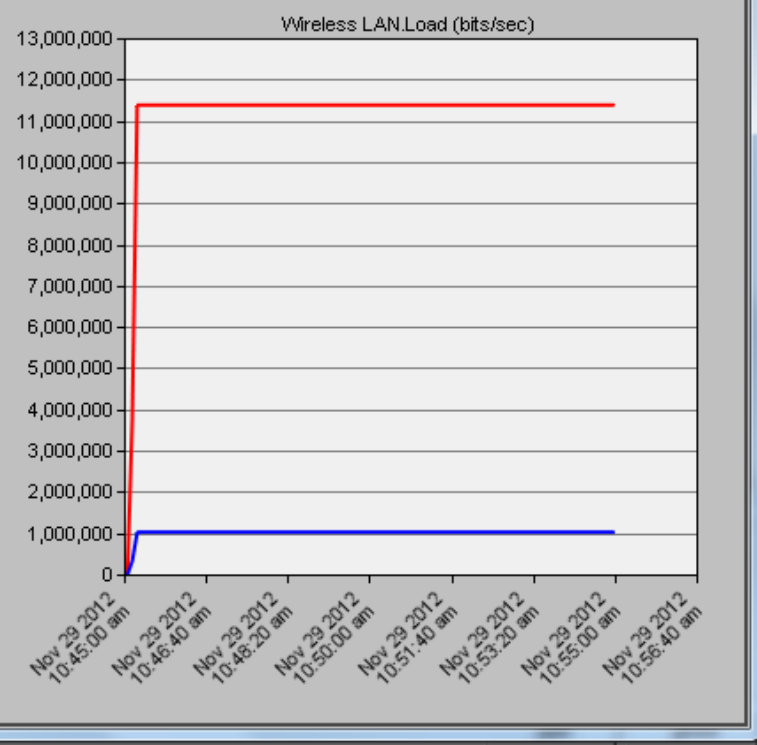

Fig.18: Load for DSSS physical layer technology at 1Mbps before (DSSS) and after (DSSS2) tuning the traffic generation parameters

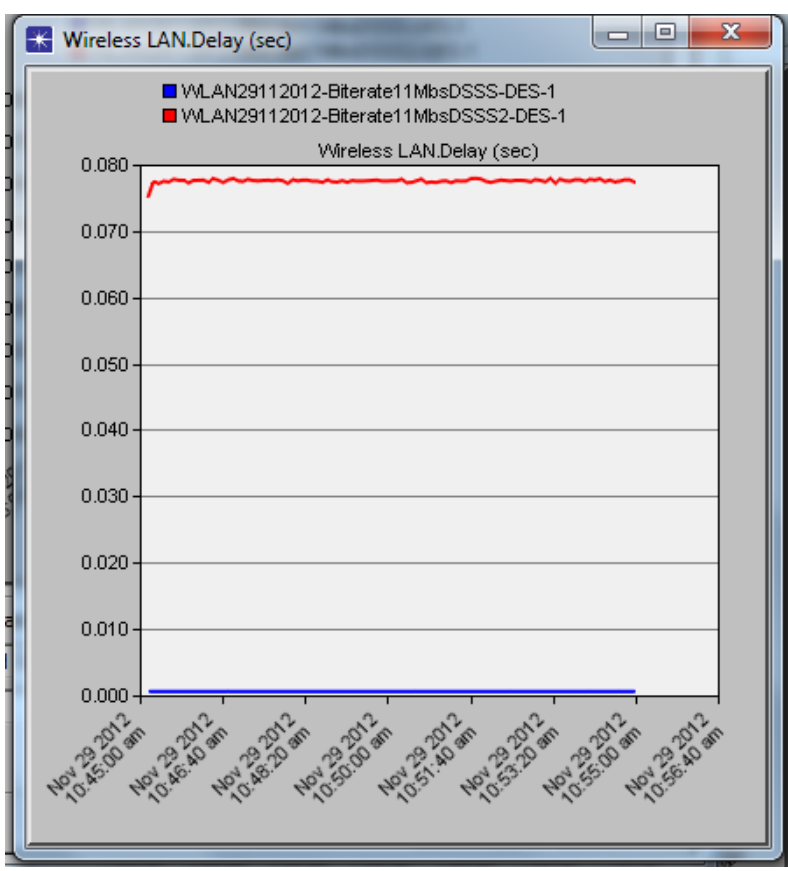

Fig.19: WLAN end to end delay for DSSS physical layer technology at 1Mbps before (DSSS) and after (DSSS2) tuning the traffic generation parameters 


\section{CONCLUSIONS}

In the proposed system, the performance of wireless network scenarios (sixteen scenarios) in OPNET Modeler 14.5 is evaluated. End to end delay, media access delay, throughput, load, traffic received and traffic sent is evaluated for the physical layer technologies IR, DSSS, FHSS and OFDM at multiple transmission rates. In terms of delay, at some physical layer technology, the delay can be improved (decreased) with increasing the transmission rate.Delay at the same transmission rate, OFDM is better than DSSS and FHSS physical layer technology. In addition, IR is better than DSSS and FHSS. To obtain better throughput, the generation of data is tuned.

\section{REFERENCES}

[1] Jeffry, R., Jackie, A. 2001 Designing a wireless network

[2] Dean A. Gratton 2007 Developing practical wireless applications
[3] Xiaodong and H. 2002 Wireless communication systems: advanced techniques for signal reception.

[4] Rahul, V. and Dr. R. K. Bansal. "Simulation \& performance analysis of wired and wireless computer networks", International Journal of Computer Applications, February 2011.

[5] Lachhman,S., Asad, Y., Malkani "Performance analysis of WLAN standards for video conferencing applications",International Journal of Wireless \& Mobile Networks (IJWMN) Vol. 3, No. 6, December 2011

[6] Rajan, R., Shipra, S. "WLAN Performance Improvisation by Fine Tuning IEEE 802.11 Parameters", International Journal of Computer Applications, April 2012.

[7] Kritika, N., Namarta. "Performance Evaluation of 802.11 WLAN Scenarios in OPNET Modeler" International Journal of Computer Applications, May 2011. 